\title{
Ambiente Domiciliar Assistido para o Monitoramento Remoto de Idosos em Ambiente Rural
}

\author{
Walkíria Garcia de Souza Silveira ${ }^{1}$, Mario Antonio Ribeiro Dantas ${ }^{1}$, \\ ${ }^{1}$ Departamento de Ciência da Computação - Universidade Federal de Juiz de Fora (UFJF) \\ Caixa Postal 20.010 - 36.016-970 - Juiz de Fora - MG - Brasil \\ \{walkiria.garcia, mario.dantas\}@ice.ufjf.br
}

\begin{abstract}
With population aging and the concern to maintain primary health care for the elderly population in rural areas, this article proposes a model for remote control of elderly people with difficult internet access, which considers edge fog and cloud to provide an appropriate structure .
\end{abstract}

Resumo. Com o envelhecimento populacional e a preocupação de manter a atenção primária à saúde da população idosa da área rural, o presente artigo propõe um modelo para o controle remoto de idosos com difícil acesso à internet, que considere edge fog e cloud para prover uma estrutura apropriada.

\section{Introdução}

O Brasil, assim como o restante do mundo, está vivenciando um envelhecimento populacional que afeta diretamente a prestação de atendimentos do SUS [Silva 2021], uma vez que essa população é a que mais faz uso de assistência médica [Penido 2018] .

Somado ao envelhecimento populacional e à preocupação com a atenção primária à saúde, temos a preocupação com a população idosa residente na zona rural, que corresponde a 15 por cento da população idosa brasileira [Penido 2018]. Devido a essa baixa porcentagem, esse grupo tem tido pouca visibilidade da sociedade, o que contribui para uma falta de informações quanto às suas necessidades, porém, estimativas indicam que existe uma maior dificuldade ao acesso à saúde primária, dado principalmente pela distância e condições do deslocamento [Garbaccio 2018].

Por outro lado, tem-se o rápido avanço tecnológico e a transformação digital, que vem trazendo contribuições aos problemas relacionados à saúde, como o ambiente domiciliar assistido (AAL), que são produtos, conceitos e serviços que tem como objetivo contribuir para uma melhor qualidade de vida dos indivíduos dentro de seu próprio ambiente domiciliar [Nakagawa et al. 2013].

Contudo, essas contribuíções e soluções são mais restritas para a população rural, devido ao limitado acesso a internet verificado no último censo agropecuário, que revelou que 28 por cento das propriedades rurais têm acesso à internet [MILANEZ 2020].

Ao observar os desafios encontrados com o envelhecimento da população e a falta de atenção aos idosos da área rural, notou-se a viabilidade de um projeto de pesquisa e desenvolvimento com ênfase no atendimento primário da população idosa residente na área rural. Com isso, o presente trabalho tem o objetivo de propor um estudo de um AAL que possa prover como um diferencial na observação das camadas de edge fog e cloud para a melhoria na vida dos idosos. 


\section{Fundamentação Teórica}

A edge computing fica localizada próximo do usuário, o que traz maior rapidez e segurança, já que os dados são processados localmente e enviados para a nuvem através de uma rede distribuída que conecta a edge com a cloud, chamada fog, que é uma tecnologia que trata dos gargalos na rede gerados pelo grande fluxo de dados coletados por dispositivos IoT. É um paradigma que completa a computação em nuvem [Habibi et al. 2020].

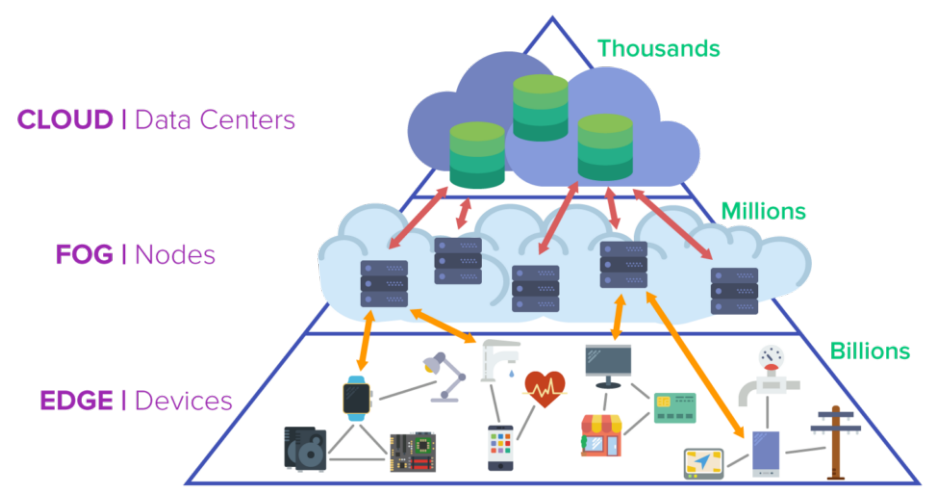

Figura 1. Edge - Fog - Cloud [Omni.sci 2021]

\section{Proposta de um AAL Rural}

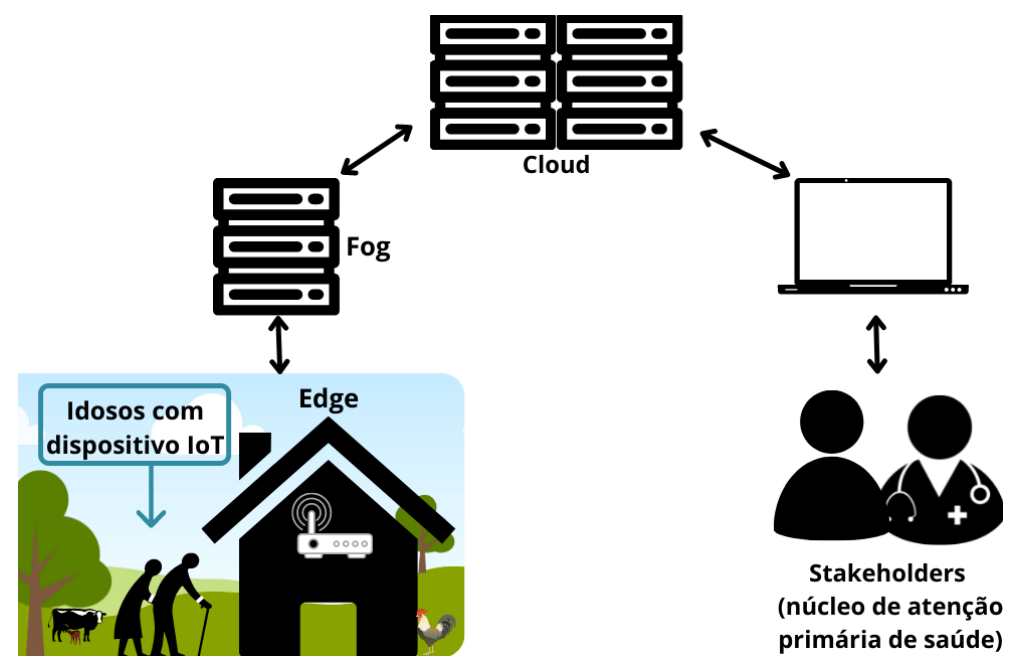

Figura 2. Modelo Proposto AAL Rural

A proposta desse estudo é desenvolver uma abordagem que considere edge fog e cloud para prover uma estrutura apropriada no controle remoto de idosos com difícil acesso à internet, em um AAL.

O AAL deve monitorar os sinais vitais do usuário através de um dispositivo usável que coleta, trata e transforma em informação esses sinais vitais, que são acessadas pelos stakeholders através de um sistema, monitorando assim a saúde do idoso e diminuindo consultas médicas.

A Figura 2 ilustra o modelo AAL rural proposto, onde temos representados os idosos moradores de áreas rurais fazendo uso frequente de um dispositivo usável. O 
objetivo é que eles compartilhem os dados coletados com um dispositivo local que faz o processamento e análises avançadas dos dados coletados. Dessa forma será realizada uma organização e higienização das informações enviando apenas o que é necessário, assim o tráfego das informações enviadas é minimizado (fog).

$\mathrm{Na}$ camada de edge pretende-se desenvolver um aplicativo para a coleta dos dados do dispositivo usável. Todos os moradores da região deverão ter um dispositivo móvel e um aplicativo. Os dados coletados pelos aplicativos serão enviados por uma rede de comunicação para a fog, que será uma aplicação servidor coletando os dados de todas as edges da região. Em nível de fog será efetuado o processamento desses dados e posteriormente o envio dos dados já processados para os servidores na nuvem (cloud).

O computador da Figura 2 representa o sistema web onde deverão ser exibidas todas as informações recebidas, é por ele que os stakeholders terão acesso às informações.

Espera-se que, com esse trabalho de pesquisa, possa-se trazer mais qualidade de vida aos idosos da área rural, realizando uma melhor atenção primária à saúde de forma eficiente e provendo uma possibilidade de aumento na qualidade de vida desse grupo.

\section{Conclusão}

O presente trabalho de pesquisa traz uma contribuição para a melhoria dos problemas relacionados à atenção primária à saúde de moradores da área rural.

O desenvolvimento do presente modelo AAL Rural deverá ser desenvolvido em duas dimensões. A primeira utilizando uma abordagem AAL e a segunda através de simulação para fazer a generalização da proposta.

\section{Referências}

Garbaccio, J. L. e. a. (2018). Aging and quality of life of elderly people in rural areas. In Revista Brasileira de Enfermagem.

Habibi, P., Farhoudi, M., Kazemian, S., Khorsandi, S., and Leon-Garcia, A. (2020). Fog computing: A comprehensive architectural survey. IEEE Access, 8:69105-69133.

MILANEZ, A. Y. e. a. (2020). Conectividade rural: situação atual e alternativas para superação da principal barreira à agricultura 4.0 no brasil = rural connectivity: current situation and alternatives to overcome the main barrier related to agriculture 4.0 in brazil. In BNDES Setorial, Rio de Janeiro,.

Nakagawa, E. Y., Antonino, P. O., Becker, M., Maldonado, J. C., Storf, H., Villela, K. B., and Rombach, D. (2013). Relevance and perspectives of aal in brazil. Journal of Systems and Software, 86(4):985-996. SI : Software Engineering in Brazil: Retrospective and Prospective Views.

Omni.sci (2021). Fog computing. https://www.omnisci.com/ technical-glossary/fog-computing. Acessado em 13/11/2021.

Penido, A. (2018). Estudo aponta que 75 por cento dos idosos usam apenas o sus. In Fiocruz.

Silva, A. M. d. M. e. a. (2021). Fragilidade entre idosos e percepção de problemas em indicadores de atributos da atenção primária à saúde: resultados do elsi-brasil. In Cadernos de Saúde Pública. 